PROCEEDINGS OF THE

AMERICAN MATHEMATICAL SOCIETY

Volume 136, Number 11, November 2008, Pages 4095-4102

S 0002-9939(08)09398-2

Article electronically published on June 11, 2008

\title{
GRADIENT ESTIMATES FOR A NONLINEAR PARABOLIC EQUATION ON RIEMANNIAN MANIFOLDS
}

\author{
YUNYAN YANG \\ (Communicated by Richard A. Wentworth)
}

\begin{abstract}
Let $(M, g)$ be a complete noncompact Riemannian manifold. In this paper, we derive a local gradient estimate for positive solutions to a simple nonlinear parabolic equation

$$
\frac{\partial u}{\partial t}=\Delta u+a u \log u+b u
$$

on $M \times[0,+\infty)$, where $a, b$ are two real constants. This equation is closely related to the gradient Ricci soliton. We extend the result of L. Ma (Journal of Functional Analysis 241 (2006) 374-382).
\end{abstract}

\section{INTRODUCTION}

Let $(M, g)$ be a complete noncompact Riemannian manifold. We consider the evolution equation

$$
\frac{\partial u}{\partial t}=\Delta u+a u \log u+b u
$$

on $M \times[0,+\infty)$, where $a, b$ are two real constants. The motivation to study equation (1.1) is to understand the Ricci flow introduced by R. Hamilton [5]. In the study of Ricci flow, one may meet the Ricci solitons, which are self-similar solutions to Ricci flow and arise in the blow-up procedure of the long time existence or convergence of the flow. Recall the following definition (see, for example, Definition 1.2.3 on page 10 of [11]).

Definition A. A Riemannian manifold $(M, g)$ is called a gradient Ricci soliton if there is a smooth function $f$ on it such that for some constant $c \in \mathbb{R}$, it satisfies

$$
R i c=c g+D^{2} f
$$

on $M$, where $D^{2} f$ is the Hessian of $f$ and Ric is the Ricci tensor of the metric $g$. If $c>0$ in $(1.2),(M, g)$ is called a shrinking soliton; if $c=0,(M, g)$ is called a steady soliton; and if $c<0,(M, g)$ is called an expanding soliton.

Suppose that $(M, g)$ is a gradient Ricci soliton and $c, f$ are described in Definition 1.1. Letting $u=e^{f}$, one can derive from (1.2) that (cf. 8], Eq. (7))

$$
\Delta u+2 c u \log u=\left(A_{0}-n c\right) u
$$

Received by the editors April 19, 2007, and, in revised form, October 13, 2007.

2000 Mathematics Subject Classification. Primary 58J05, 58J35.

The author was supported in part by the NSFC 10601065 . 
for some constant $A_{0}$. Equation (1.3) is a nonlinear elliptic equation and is a special case of equation (1.1). For this kind of equation, L. Ma (see Theorem 1 in 8]) proved the following result.

Theorem B (L. Ma). Let $(M, g)$ be a complete noncompact Riemannian manifold of dimension $n$ with Ricci curvature bounded below by the constant $-K:=-K(2 R)$, where $R>0$ and $K(2 R) \geq 0$, in the geodesic ball $B_{p}(2 R)$ for some fixed point $p \in M$. Let $u$ be a positive smooth solution to the elliptic equation

$$
\Delta u+a u \log u=0 \quad \text { in } \quad M,
$$

where $a<0$ is a real constant. Let $f=\log u$ and $(f, 2 f)$ be the maximum among $f$ and $2 f$. Then there are two uniform positive constants $c_{1}$ and $c_{2}$ such that

$$
|\nabla f|^{2}+a(f, 2 f) \leq \frac{n\left((n+2) c_{1}^{2}+(n-1) c_{1}^{2}(1+R \sqrt{K})+c_{2}\right)}{R^{2}}+2 n(K-a)
$$

on $B_{p}(R)$.

It was pointed out by L. Ma $[8$ that it would be interesting to consider the local gradient estimate for positive solutions to the evolution equation (1.1). We shall give an answer to this question in this paper. Our main result is the following:

Theorem 1.1. Let $M$ be a complete noncompact Riemannian manifold of dimension $n$ without boundary. Suppose the Ricci curvature of $M$ is bounded below by the constant $-K(2 R)$, where $R>0$ and $K(2 R) \geq 0$, in the geodesic ball $B_{p}(2 R)$ with radius $2 R$ around $p \in M$. If $u(x, t)$ is a positive smooth solution to equation (1.1) on $M \times[0,+\infty)$, let $f(x, t)=\log u(x, t)$. Then

(i) if $a<0$, we have for any $\alpha>1$ and $0<\delta<1$,

$$
\begin{gathered}
|\nabla f|^{2}(x, t)+\alpha a f(x, t)+\alpha b-\alpha f_{t}(x, t) \leq \frac{n \alpha^{2}}{2 \delta t}+\frac{n \alpha^{2}}{2 \delta}\left\{\frac{2 \epsilon^{2}}{R^{2}}+\frac{\nu}{R^{2}}-\frac{a}{2}\right. \\
\left.+\frac{\epsilon^{2}}{R^{2}}(n-1)(1+R \sqrt{K(2 R)})+\frac{K(2 R)}{\alpha-1}+\frac{n \alpha^{2} \epsilon^{2}}{8(1-\delta)(\alpha-1) R^{2}}\right\}
\end{gathered}
$$

on $B_{p}(R) \times(0,+\infty)$, where $\epsilon>0$ and $\nu>0$ are some constants;

(ii) if $a>0$, we have for any $\alpha>1$ and $0<\delta<1$,

$$
\begin{array}{r}
|\nabla f|^{2}(x, t)+\alpha a f(x, t)+\alpha b-\alpha f_{t}(x, t) \leq \frac{n \alpha^{2}}{2 \delta t}+\frac{n \alpha^{2}}{2 \delta}\left\{\frac{2 \epsilon^{2}}{R^{2}}+\frac{\nu}{R^{2}}+a\right. \\
\left.+\frac{\epsilon^{2}}{R^{2}}(n-1)(1+R \sqrt{K(2 R)})+\frac{K(2 R)}{\alpha-1}+\frac{n \alpha^{2} \epsilon^{2}}{8(1-\delta)(\alpha-1) R^{2}}\right\}
\end{array}
$$

on $B_{p}(R) \times(0,+\infty)$, where $\epsilon>0$ and $\nu>0$ are some constants.

It can be checked that the estimate in (i) of Theorem 1.1 is better than Theorem $\mathrm{B}$ in the case that $K(2 R)=0, b=0$ and $u(x, t)$ is independent of $t$. An interesting corollary of Theorem 1.1 is the following result.

Corollary 1.2. Let $M$ be a complete noncompact Riemannian manifold of dimension $n$ without boundary. Suppose that the Ricci curvature of $M$ is nonnegative. If $u(x)$ is a positive smooth solution to the equation

$$
\Delta u+a u \log u=0 \quad \text { on } \quad M,
$$


then if $a<0$, we have $u(x) \geq e^{-n / 4}$ for all $x \in M$; and if $a>0$, we have $u(x) \leq e^{n / 2}$ for all $x \in M$.

Our method of proving Theorem 1.1 is the gradient estimates, which originated first in Yau 12 (see also Cheng-Yau [4]), and was developed by Li-Yau 7], Li 6], Negrin [10, Melas 9] and Asserda [1. However, one may notice that there are not many results on gradient estimates for nonlinear equations on complete noncompact Riemannian manifolds (cf. Li [6]). In the rest of the paper, we will prove Theorem 1.1 (Sections 2, 3) and Corollary 1.2 (Section 4).

\section{THE CASE $a<0$}

In this section, we derive gradient estimates on positive smooth solutions of the equation (1.1) with $a<0$. Replacing $u$ by $e^{b / a} u$, we only need to consider positive smooth solutions of the following equation:

$$
\frac{\partial u}{\partial t}=\Delta u+a u \log u
$$

on $M \times[0,+\infty)$. Recall the well-known Weitzenbock-Bochner formula [2]:

Lemma 2.1. Given any smooth function $h$ on $(M, g)$, it holds that

$$
\Delta|\nabla h|^{2}=2 \nabla h \nabla \Delta h+2\left|D^{2} h\right|^{2}+2 R i c(\nabla h, \nabla h),
$$

where $D^{2} h$ is the Hessian of $h$ and Ric is the Ricci tensor of the metric $g$.

Let $u(x, t)$ be a positive smooth solution of $(2.1)$ on $M \times[0,+\infty)$. Define two functions on $M \times[0,+\infty)$ as

$$
\begin{aligned}
& f(x, t)=\log u(x, t), \\
& F(x, t)=t\left\{|\nabla f|^{2}(x, t)+\alpha a f(x, t)-\alpha f_{t}(x, t)\right\},
\end{aligned}
$$

where $\alpha>1$ is a constant. Then we have by (2.1),

$$
\begin{aligned}
\Delta f & =f_{t}-a f-|\nabla f|^{2} \\
& =-\frac{F}{\alpha t}-\left(1-\frac{1}{\alpha}\right)|\nabla f|^{2} .
\end{aligned}
$$

It follows that

$$
f_{t t}=\Delta f_{t}+a f_{t}+2 \nabla f \nabla f_{t}
$$

From the definition of $F(x, t)$, we know that

$$
|\nabla f|^{2}=\frac{F}{t}-\alpha a f+\alpha f_{t}
$$


Using (2.2), (2.3) and (2.4), a delicate but straightforward calculation gives

$$
\begin{aligned}
\Delta F= & t\left\{\Delta|\nabla f|^{2}+\alpha a \Delta f-\alpha \Delta f_{t}\right\} \\
= & t\left\{2 \nabla f \nabla \Delta f+2\left|D^{2} f\right|^{2}+2 \operatorname{Ric}(\nabla f, \nabla f)\right. \\
& -\frac{\alpha}{t} a F+\alpha(\alpha-1) a^{2} f-\alpha(\alpha-1) a f_{t} \\
& \left.-\alpha\left(f_{t t}-a f_{t}-2 \nabla f \nabla f_{t}\right)\right\} \\
= & t\left\{-\frac{2}{t} \nabla f \nabla F+2(\alpha-1) a|\nabla f|^{2}+2 \nabla f \nabla f_{t}\right. \\
& +2\left|D^{2} f\right|^{2}+2 \operatorname{Ric}(\nabla f, \nabla f) \\
& \left.-\frac{\alpha}{t} a F+\alpha(\alpha-1) a^{2} f-\alpha(\alpha-2) a f_{t}-\alpha f_{t t}\right\}
\end{aligned}
$$

and

$$
\begin{aligned}
F_{t} & =\left\{|\nabla f|^{2}+\alpha a f-\alpha f_{t}\right\}+t\left\{2 \nabla f \nabla f_{t}+\alpha a f_{t}-\alpha f_{t t}\right\} \\
& =\frac{F}{t}+t\left\{2 \nabla f \nabla f_{t}+\alpha a f_{t}-\alpha f_{t t}\right\} .
\end{aligned}
$$

Combining (2.5) and (2.6), using (2.3) and the fact that $\left|D^{2} f\right|^{2} \geq|\Delta f|^{2} / n$, we obtain

$$
\begin{aligned}
\left(\Delta-\frac{\partial}{\partial t}\right) F= & -2 \nabla f \nabla F+(\alpha-1) a t|\nabla f|^{2}-a F \\
& -\frac{F}{t}+2 t\left|D^{2} f\right|^{2}+2 t \operatorname{Ric}(\nabla f, \nabla f) \\
\geq & -2 \nabla f \nabla F+t\left\{\frac{2}{n}\left(-\frac{F}{\alpha t}-\left(1-\frac{1}{\alpha}\right)|\nabla f|^{2}\right)^{2}\right. \\
& \left.+((\alpha-1) a-2 K(2 R))|\nabla f|^{2}\right\}-a F-\frac{F}{t}
\end{aligned}
$$

on $B_{p}(2 R) \times[0, \infty)$, where $B_{p}(2 R) \subset M$ denotes the geodesic ball with radius $2 R$ around $p \in M$. Here we have used the assumption that $\operatorname{Ric}(\nabla f, \nabla f) \geq$ $-K(2 R)|\nabla f|^{2}$ on $B_{p}(2 R)$.

Let $\chi \in C^{2}[0, \infty)$ be a cut-off function such that $\chi(r)=1$ for $r \leq 1, \chi(r)=0$ for $r \geq 2$, and $0 \leq \chi(r) \leq 1$. In addition one can choose $\chi$ such that $\chi^{\prime}(r) \geq-\epsilon \chi^{1 / 2}(r)$, and $\chi^{\prime \prime}(r) \geq-\nu$, where $\epsilon$ and $\nu$ are some positive constants. Denote by $d(p, x)$ the geodesic distance between $p$ and $x$. Set

$$
\phi(x)=\chi\left(\frac{d(p, x)}{R}\right) .
$$

Using an argument of Calabi [3] (see also Cheng and Yau [4]), we can assume, without loss of generality, that $\phi(x) \in C^{2}(M)$ with support in $B_{p}(2 R)$. Clearly we have

$$
|\nabla \phi|^{2} \leq \frac{\epsilon^{2}}{R^{2}} \phi
$$

Using the Laplacian comparison theorem (cf. 2], Theorem 5.13), we obtain (see also [6, 10, 8])

$$
\Delta \phi \geq-\frac{\epsilon^{2}}{R^{2}}(n-1)(1+R \sqrt{K(2 R)})-\frac{\nu}{R^{2}}
$$


For any fixed $T>0, \sup _{(x, t) \in B_{p}(2 R) \times[0, T]} \phi(x) F(x, t)$ must be attained at some point $(z, s) \in B_{p}(2 R) \times[0, T]$. Without loss of generality, we can assume

$$
\lambda:=\sup _{(x, t) \in B_{p}(2 R) \times[0, T]} \phi(x) F(x, t)>0,
$$

for otherwise $\lambda \leq 0$. (1.5) is already true on $B_{p}(2 R) \times(0, T]$; here $b=0$.

At the point $(z, s)$, we have

$$
\nabla(\phi F)=0, \quad \phi \Delta(\phi F) \leq 0, \quad F_{t} \geq 0 .
$$

It follows that

$$
\phi \Delta F+F \Delta \phi-2 F|\nabla \phi|^{2} \phi^{-1} \leq 0
$$

at $(z, s)$. This inequality together with the estimates (2.8) and (2.9) yields

$$
\phi \Delta F \leq\left(\frac{2 \epsilon^{2}}{R^{2}}+\frac{\epsilon^{2}}{R^{2}}(n-1)(1+R \sqrt{K(2 R)})+\frac{\nu}{R^{2}}\right) F
$$

at $(z, s)$. Multiplying both sides of (2.7) by $\phi$, we have by (2.10) that

$$
\begin{aligned}
(2.12) \phi \Delta F \geq & -\frac{2 \epsilon}{R} \phi^{\frac{1}{2}} F|\nabla f|-a \phi F-\frac{1}{s} \phi F+s \phi\left\{\frac{2}{n}\left(\frac{F}{\alpha s}+\frac{\alpha-1}{\alpha}|\nabla f|^{2}\right)^{2}\right. \\
& \left.+((\alpha-1) a-2 K(2 R))|\nabla f|^{2}\right\}
\end{aligned}
$$

at $(z, s)$. Following E. Negrin [10, we denote

$$
\mu=|\nabla f|^{2}(z, s) / F(z, s) .
$$

For simplicity, set

$$
B=\frac{2 \epsilon^{2}}{R^{2}}+\frac{\epsilon^{2}}{R^{2}}(n-1)(1+R \sqrt{K(2 R)})+\frac{\nu}{R^{2}} .
$$

By (2.11) and (2.12), we have at $(z, s)$,

$$
\begin{aligned}
& B F+\frac{2 \epsilon}{R} \phi^{\frac{1}{2}} F^{\frac{3}{2}} \mu^{\frac{1}{2}}+a \phi F+\frac{1}{s} \phi F \\
& \geq s \phi\left\{\frac{2}{n} F^{2}\left(\frac{1+(\alpha-1) \mu s}{\alpha s}\right)^{2}+((\alpha-1) a-2 K(2 R)) F \mu\right\} .
\end{aligned}
$$

Multiplying both sides of the above inequality by $s \phi$ and using the fact that $0 \leq$ $\phi \leq 1$ and $a<0$, we obtain at $(z, s)$,

$$
\begin{aligned}
& B s \phi F+\frac{2 \epsilon}{R}(\phi F)^{\frac{3}{2}} \mu^{\frac{1}{2}} s+\phi F \\
& \geq \frac{2}{n \alpha^{2}}(\phi F)^{2}(1+(\alpha-1) \mu s)^{2}+((\alpha-1) a-2 K(2 R)) \phi F \mu s^{2} .
\end{aligned}
$$

For any fixed $0<\delta<1$, at $(z, s)$

$$
\begin{aligned}
\frac{2 \epsilon}{R}(\phi F)^{\frac{3}{2}} \mu^{\frac{1}{2}} s \leq & (1-\delta) \frac{2}{n \alpha^{2}}(\phi F)^{2}(1+(\alpha-1) \mu s)^{2} \\
& +\frac{n \alpha^{2} \phi F \mu s^{2} \epsilon^{2} / R^{2}}{2(1-\delta)(1+(\alpha-1) \mu s)^{2}}
\end{aligned}
$$

Using the notation $\lambda=\phi(z) F(z, s)$, we have by (2.13) and (2.14),

$$
\begin{aligned}
& B s \lambda+\frac{n \alpha^{2} \mu s^{2} \epsilon^{2} / R^{2}}{2(1-\delta)(1+(\alpha-1) \mu s)^{2}} \lambda+\lambda \\
& \geq \frac{2 \delta}{n \alpha^{2}}(1+(\alpha-1) \mu s)^{2} \lambda^{2}+((\alpha-1) a-2 K(2 R)) \mu s^{2} \lambda .
\end{aligned}
$$


Since $\lambda>0$, we have by (2.15),

$$
\begin{aligned}
\lambda \leq \frac{n \alpha^{2}}{2 \delta} \frac{1}{(1+(\alpha-1) \mu s)^{2}}\{B s & +\frac{n \alpha^{2} \mu s^{2} \epsilon^{2} / R^{2}}{2(1-\delta)(1+(\alpha-1) \mu s)^{2}} \\
& \left.+1+((\alpha-1)|a|+2 K(2 R)) \mu s^{2}\right\} .
\end{aligned}
$$

The inequalities

$$
\begin{aligned}
\frac{1+((\alpha-1)|a|+2 K(2 R)) \mu s^{2}}{(1+(\alpha-1) \mu s)^{2}} & \leq \frac{1+((\alpha-1)|a|+2 K(2 R)) s \cdot \mu s}{1+2(\alpha-1) \mu s} \\
& \leq 1+\frac{(\alpha-1)|a|+2 K(2 R)}{2(\alpha-1)} s
\end{aligned}
$$

and

$$
\frac{n \alpha^{2} \mu s^{2} \epsilon^{2} / R^{2}}{2(1-\delta)(1+(\alpha-1) \mu s)^{4}} \leq \frac{n \alpha^{2} \epsilon^{2}}{8(1-\delta)(\alpha-1) R^{2}} s,
$$

together with (2.16), give

$$
\lambda \leq \frac{n \alpha^{2}}{2 \delta}\left\{B s+1+\frac{(\alpha-1)|a|+2 K(2 R)}{2(\alpha-1)} s+\frac{n \alpha^{2} \epsilon^{2}}{8(1-\delta)(\alpha-1) R^{2}} s\right\} .
$$

Notice that for all $t \in[0, T]$,

$$
\sup _{B_{p}(R)} t\left\{|\nabla f|^{2}+\alpha a f-\alpha f_{t}\right\} \leq \lambda=\phi(z) F(z, s) .
$$

Combining (2.17) and (2.18), we have

$$
\begin{aligned}
\sup _{x \in B_{p}(R)} & \left\{|\nabla f|^{2}(x, T)+\alpha a f(x, T)-\alpha f_{t}(x, T)\right\} \leq \frac{n \alpha^{2}}{2 \delta T} \\
+ & \frac{n \alpha^{2}}{2 \delta}\left\{B-\frac{a}{2}+\frac{K(2 R)}{\alpha-1}+\frac{n \alpha^{2} \epsilon^{2}}{8(1-\delta)(\alpha-1) R^{2}}\right\} .
\end{aligned}
$$

Now (i) of Theorem 1.1 follows easily from (2.19) since $T>0$ is arbitrary.

\section{THE CASE $a>0$}

In this section, we prove (ii) of Theorem 1.1. Also, we only need to consider positive solutions of (2.1). We adopt the same notation as in Section 2, but here $a>0$. Without loss of generality, we can assume $\phi F$ achieves its positive maximum at $(z, s) \in B_{p}(2 R) \times[0, T]$. Using a similar means of deriving (2.13), we obtain the following estimate at point $(z, s)$ :

$$
\begin{aligned}
& B s \phi F+\frac{2 \epsilon}{R}(\phi F)^{\frac{3}{2}} \mu^{\frac{1}{2}} s+a s \phi F+\phi F \\
& \geq \frac{2}{n \alpha^{2}}(\phi F)^{2}(1+(\alpha-1) \mu s)^{2}-2 K(2 R) \phi F \mu s^{2} .
\end{aligned}
$$

Denote $\lambda=\phi(z) F(z, s)$. By (2.14), the inequality (3.1) becomes

$$
\begin{aligned}
& B s \lambda+\frac{n \alpha^{2} \mu s^{2} \epsilon^{2} / R^{2}}{2(1-\delta)(1+(\alpha-1) \mu s)^{2}} \lambda+a s \lambda+\lambda \\
& \geq \frac{2 \delta}{n \alpha^{2}}(1+(\alpha-1) \mu s)^{2} \lambda^{2}-2 K(2 R) \mu s^{2} \lambda .
\end{aligned}
$$


Note that for $\lambda>0$, dividing both sides of (3.2) by $\lambda$, we have

$$
\begin{aligned}
\lambda \leq & \frac{n \alpha^{2}}{2 \delta} \frac{1}{(1+(\alpha-1) \mu s)^{2}}\left\{B s+\frac{n \alpha^{2} \mu s^{2} \epsilon^{2} / R^{2}}{2(1-\delta)(1+(\alpha-1) \mu s)^{2}}\right. \\
& \left.+a s+1+2 K(2 R) \mu s^{2}\right\} \\
\leq & \frac{n \alpha^{2}}{2 \delta}\left\{B s+\frac{n \alpha^{2} \epsilon^{2}}{8(1-\delta)(\alpha-1) R^{2}} s+a s+1+\frac{K(2 R)}{\alpha-1} s\right\} .
\end{aligned}
$$

With the same method of deriving (2.19), one can get

$$
\begin{aligned}
\sup _{x \in B_{p}(R)} & \left\{|\nabla f|^{2}(x, T)+\alpha a f(x, T)-\alpha f_{t}(x, T)\right\} \leq \frac{n \alpha^{2}}{2 \delta T} \\
+ & \frac{n \alpha^{2}}{2 \delta}\left\{B+a+\frac{K(2 R)}{\alpha-1}+\frac{n \alpha^{2} \epsilon^{2}}{8(1-\delta)(\alpha-1) R^{2}}\right\} .
\end{aligned}
$$

Now (ii) of Theorem 1.1 follows easily from (3.4) since $T>0$ is arbitrary.

\section{Proof of Corollary 1.2}

Proof of Corollary 1.2. Suppose $u$ is a positive smooth solution to the equation

$$
\Delta u+a u \log u=0 \quad \text { on } \quad M .
$$

Fix a point $p \in M$. Let $B_{p}(R)$ be a geodesic ball with radius $R>0$ around $p$.

Case 1. $a<0$.

Since the Ricci curvature of $M$ is nonnegative and $u(x)$ is independent of $t$, by (i) of Theorem 1.1, we have on $B_{p}(R)$,

$$
\begin{aligned}
\frac{|\nabla u|^{2}}{u^{2}} & +\alpha a \log u(x) \leq \frac{n \alpha^{2}}{2 \delta}\left\{\frac{2 \epsilon^{2}}{R^{2}}+\frac{\nu}{R^{2}}-\frac{a}{2}\right. \\
& \left.+\frac{\epsilon^{2}}{R^{2}}(n-1)+\frac{n \alpha^{2} \epsilon^{2}}{8(1-\delta)(\alpha-1) R^{2}}\right\},
\end{aligned}
$$

where $\alpha>1$ and $0<\delta<1$ are two arbitrary real constants, and $\epsilon>0$ and $\nu>0$ are some fixed constants. Letting $R \rightarrow+\infty$, we have by (4.1),

$$
\alpha a \log u(x) \leq-\frac{n \alpha^{2} a}{4 \delta} .
$$

Since $a<0$, letting $\alpha \rightarrow 1$ and $\delta \rightarrow 1$, we have by (4.2),

$$
u(x) \geq e^{-n / 4} \quad \text { for all } \quad x \in M .
$$

Case 2. $a>0$.

Using the same method of deriving (4.2), we have by (ii) of Theorem 1.1,

$$
\alpha a \log u(x) \leq \frac{n \alpha^{2} a}{2 \delta} .
$$

Since $a>0$, letting $\alpha \rightarrow 1$ and $\delta \rightarrow 1$, we have by (4.4),

$$
u(x) \leq e^{n / 2} \text { for all } x \in M,
$$

and whence we have proved Corollary 1.2. 


\section{REFERENCES}

[1] S. Asserda, A Liouville theorem for the Schrödinger operator with drift, C. R. Acad. Sci. Paris, Ser. I, 342 (2006), 393-398. MR2209217 (2007d:58027)

[2] T. Aubin, Nonlinear Analysis on Manifolds, Springer, New York, 1982. MR681859 (85j:58002)

[3] E. Calabi, An extension of E. Hopf's maximum principle with an application to Riemannian geometry, Duke Math. J., 25 (1958), 45-56. MR0092069 (19:1056e)

[4] S. Y. Cheng and S. T. Yau, Differential equations on Riemannian manifolds and their geometric applications, Comm. Pure Appl. Math., 28 (1975), 333-354. MR0385749 (52:6608)

[5] R. Hamilton, Three-manifolds with positive Ricci curvature, J. Diff. Geom., 17 (1982), 255306. MR664497 (84a:53050)

[6] J. Li, Gradient estimates and Harnack inequalities for nonlinear parabolic and nonlinear elliptic equations on Riemannian manifolds, J. Funct. Anal., 100 (1991), 233-256. MR.1125225 (92k:58257)

[7] P. Li and S. T. Yau, On the parabolic kernel of the Schrödinger operator, Acta Math., 156 (1986), 153-201. MR834612 (87f:58156)

[8] L. Ma, Gradient estimates for a simple elliptic equation on non-compact Riemannian manifolds, J. Funct. Anal., 241 (2006), 374-382. MR2264255 (2007e:53034)

[9] A. Melas, A Liouville type theorem for the Schrödinger operator, Proc. Amer. Math. Soc., 127 (1999), 3353-3359. MR.1623036 (2000d:58034)

[10] E. Negrin, Gradient estimates and a Liouville type theorem for the Schrödinger operator, J. Funct. Anal., 127 (1995), 198-203. MR1308622 (96a:58175)

[11] P. Topping, Lectures on the Ricci Flow, London Math. Soc. Lect. Note Ser., 325, Cambridge Univ. Press, Cambridge, 2006. MR2265040 (2007h:53105)

[12] S. T. Yau, Harmonic functions on complete Riemannian manifolds, Comm. Pure Appl. Math., 28 (1975), 201-228. MR0431040(55:4042)

Department of Mathematics, Information School, Renmin University of China, Beijing 100872, People's Republic of China

E-mail address: yunyanyang@ruc.edu.cn 